\title{
An Assessment of Losses in the Field Coil of the Compulsator under Dynamic Conditions
}

\author{
Siddharth B. PRATAP and Mircea D. DRIGA
}

\begin{abstract}
Air core compensated pulsed alternators are being developed as compact power supplies for tactical electromagnetic gun systems. The field coil of the compensated pulsed alternator is a critical component that establishes the excitation magnetic field. Since the machine is air cored the number of ampere-turns required from the field coil are significant, especially considering that the excitation flux density near the armature is typically in the range of about $3 \mathrm{~T}$ to $4 \mathrm{~T}$ and that near the field conductors in the range from $7 \mathrm{~T}$ to $10 \mathrm{~T}$. The rotating nature of the field coil requires that it be light so that it can be supported under centrifugal loads. This implies that the current density in the field coil conductors is quite large. One way of overcoming the excessive heating associated with the high current density is to charge the field coil fast (in tens of milliseconds). However charging the field coil too fast also results in transient losses due too proximity and skin effects. These must be accounted for in the design and simulation of these machines.

During the discharge into the load, transient currents flow in the armature winding. These currents produce magnetic fields that interact with the field coils and produce additional losses. This calculation is complicated by the fact that there is relative motion between the conductors.
\end{abstract}

This paper describes a two dimensional numerical analysis that has been conducted to evaluate the losses in the field coil under these two dynamic conditions. The method integrates circuit analysis with finite element calculations. This approach is essential because the field coil conductors have a connectivity that must be described in the calculations. The motional aspects have been accounted for by choosing a reference frame on the rotor and carrying out the calculations in the d-q frame attached to the field coil.

The results of this analysis are presented, along with the means of optimizing the field coil and machine geometry in view of the transient losses.

\section{Problem Definition}

$\mathrm{F}$ IG. 1 shows a typical cross section through the compulsator showing only the windings. To capture the

Manuscript received January 9, 2002. A portion of this research was carried out under the Electric Gun Program sponsored by the U.S. Army Research Laboratory through Lockheed-Martin Missile and Fire Control under contract number 4300050944.

S.B. Pratap is affiliated with The University of Texas at Austin Center for Electromechanics, Austin, Texas 78758, telephone (512) 471-4496, fax (512) 471-0781.

M.D. Driga is affiliated with The University of Texas at Austin Department of Electrical and Computer Engineering, Austin, Texas 78712, telephone (512) 471-6193, fax (512) 471-3652. details, only one pole of a four pole machine is shown. The machine consists of a field coil on the rotor that generates the excitation field and four armature phases on the stator which generate the high power. The shield is present so as to contain the magnetic fields within a small volume. For structural and thermal purposes it is desirable that the field coil conductors be made of solid aluminum conductors, and not out of litz wire. Making the field coil out of litz wire would minimize the effects of the transient magnetic fields on heat losses; however, the reduced cross section (due to poor packing fraction), and lower modulus of the litz wire would make it difficult to implement. Having decided to use solid aluminum conductors for the field coil, one must now accurately account for all the heat dissipation in it accurately.

The field coil experiences transient magnetic fields and therefore eddy current losses throughout the operational cycle of the compulsator. First the field coil charges up from zero to some maximum field current in a short time (typically in tens of milliseconds) and then, during the discharge, it interacts with the magnetic fields produced by the armature windings. The latter interaction is complicated by the fact that there is relative motion between the windings. This paper describes a method of assessing these losses in the field coil during a typical launch sequence.

\section{ANALYTICAL TOOL}

The analytical tool described here uses a combination of finite element and circuit analysis techniques [1] to arrive at an estimate of the transient losses. Finite element tools that utilize relative motion terms (convective diffusion equation) usually have difficulty arriving at solutions, especially when the relative motion increases above a few $100 \mathrm{~m} / \mathrm{s}$. Some methods have been devised that do force a solution (e.g. upwinding); however, the accuracy remains questionable [2]. In the present approach, the reference frame has been moved to the rotor, thereby eliminating the need for the convective diffusion term in solving for the magnetic field distribution. This technique of referring terms to the rotor frame of reference is not new and has been utilized historically in analyzing electric machinery [3]. The reference coordinates in the rotor frame of reference are called the $\mathrm{d}$-q axes. The d-axis is aligned with the magnetic axis of the rotor field and the q-axis is perpendicular to it, as shown in Fig. 2.

Applying these transformation techniques [4] to the reference axes on the rotor, one obtains a set of windings that 
are stationary with respect to each other as shown in figure 3 , however the currents that they carry are altered in accordance with the transformations to the rotor axes. For example in a typical launch one would obtain four measurable-phase currents as shown in Fig. 4a and after transformation to the $\mathrm{d}-\mathrm{q}$ axes on the rotor the currents would appear as shown in Fig. 4b. These currents would correspond to the currents that are flowing in the stator coils of layers 1 and 2 (Fig. 3). It is to be noted that these currents are not measurable; they are simply an artifact of the transformation. Note how the q-axis currents reverse directions during reclamation of gun and field coil energy. This is to be expected as the torque on the machine is reversed during these two processes. Combining the effects of both these windings (layers 1 and 2), the effective magnetic field axis of the stator would traverse a locus shown in Fig. 5 with respect to the $d-q$ axes on the rotor. This oscillation of the magnetic field produced by the stator windings is a source of additional losses in the field coil.

These transformed stator currents form the input to the stator coils in the finite element model and are obtained from an independent circuit simulation of the entire system. The transformations mentioned above apply to each spatial harmonic separately. Thus the above transformation process is repeated for the third spatial harmonic also. The third spatial harmonic is included in the model as a 'd' and ' $\mathrm{q}$ ' axis 12-pole stator winding. The locus of the third harmonic magnetic axis is a set of concentric circles (varying amplitude) rotating at four times the electrical frequency with respect to the rotor.

An additional input is required for the analysis as shown in Fig. 2 (circuit), i.e., the applied voltage to the field coil during self-excitation and field reclamation. During the gun discharge the field coil freewheels and the applied voltage is zero. This applied voltage is also obtained from the independent circuit simulation.

Note that the current in the field coil or the compensating winding is not specified. These will be obtained as responses from the finite element model. However, given the above excitations that are input to the finite element code, if the transformations and models are valid then the finite element code will give the same currents in the rotor coils as the independent circuit simulations. Thus, this constitutes a check on the model, as it confirms that in the finite element model we have created the correct transient magnetic fields on the rotor, and that the lumped parameter approach gives the same results as the field approach. The match between these two methods is excellent, and is shown in Figs. 6 and 7 for the two rotor coils. This same check was repeated with a compulsator design without a compensating winding and there, too, the match was excellent.

The finite element code models only one pole of the machine and uses asymmetric boundary conditions at the edges of the pole. A connectivity matrix provides the finite element code with the information about how the different conductors on the rotor are connected to each other. It also constrains the current in each series connected conductor on the rotor to have the identical total currents. The distribution of this current is, of course, governed by the local magnetic fields and their variations with time.

This analytical tool then calculates the current distribution in the conductors. From this information, one can obtain the power loss in the conductors and the energy dissipation. Fig. 8 is an example of the finite element model.

\section{RESULTS}

Having developed an analytical tool to assess the transient losses in the field coil and having established its validity, the next step is to evaluate the losses in the field coil under different charging times, different field coil geometries and also testing the effectiveness of the compensating winding.

Effect of charging rates on transient losses

The first study that was conducted evaluated how increasing the charging rates would change the losses. It is clear that the faster charging scheme will reduce the losses because the action integral ( $\left.\int i^{2} d t\right)$ to the same current level is lower. However the transient effects are stronger with the faster charging rate and this could overcome the benefits of the lower action. A field coil geometry similar to the one shown in figure 1 was used. Charging times were changed between $12.5 \mathrm{~ms}$ and $25 \mathrm{~ms}$. A charging time of less than 10 milliseconds is possible but not achievable practically because of the size of the converter to do this task and other design constraints. The general conclusions from this investigation were that the ratio of transient losses to dc losses was higher for the faster charging rates as was expected. However these effects were not sufficient to overcome the effects of the lower action. Thus faster charging rates continued to give increased efficiencies in the range of charging times investigated.

\section{INVESTIGATING THE ENTIRE OPERATING SEQUENCE}

The previous study looked at only the initial charging times. This investigation looked at the entire sequence, i.e., field charging, gun discharge and gun energy reclamation, and also field energy reclamation. The first investigation considered a compensated design. This machine had a field coil layout similar to the one shown in Fig. 1. Then the same machine was analyzed with the compensating winding disabled. This evaluates the beneficial shielding effects that the compensating winding has on the field coil. Then the field coil geometry was altered to that shown in Fig. 9. Here the conductors are arranged in two layers and are wider circumferentially as compared to the geometry of Fig. 1.

Clearly, during the initial charging and the reclaiming at the end of the launch the largest magnetic fields are produced locally by the field coil itself. The currents in the armature are low enough that it does not produce significant fields near the field coil during these times. In table 1, two loss ratios for identical rates with the compensated and the uncompensated machines are compared and are, in fact, the same. However, when comparing the loss ratios during the discharge one sees a 
significant difference. This can be explained by the fact that the compensating winding shields the field coil from the quadrature fields in the compensated design; therefore, the compensated design has a lower loss ratio. The compensating winding thus helps to reduce losses in the field coil; however, it is not clear that it would help the overall efficiency, due to the losses in the compensating coil itself. To reap the benefits of efficiency, the compensating winding resistance must be low (thicker conductors). The introduction of the compensating winding itself, however, pushes the field winding radially deeper into the rotor. This in turn reduces the coupling with the armature and therefore needs more ampereturns, and more losses. So the decision to introduce the compensating winding is complicated and must be evaluated for each design separately. There are other factors (external to the machine) which also govern this decision.

The field coil design of Fig. 9 was evaluated without the compensating winding. Comparing it with the second row in table 1 , one sees that the loss ratios for all the events is higher, clearly indicating that Fig. 9 would not be preferred as design option for the field coil. The reason for the higher losses is that the conductors represent a larger cross-section to the predominantly radial flux passing through that region. Figs. 10 and 11 give a comparison of the current density in the conductors at the same instant in time, i.e., when the charging cycle is near completion.

\section{CONCLUSIONS}

An analytical tool has been developed which helps to assess the transient losses in the field coils of the compulsator. Transformation to the rotor frame of reference allows problem to be solved without the convective diffusion term thus simplifying the formulation considerably. The accuracy of the finite element model has been confirmed by comparing the responses of the rotor circuits to independent circuit simulations. This tool was then used to evaluate the effect that the charging rates will have on the losses in the field coil and also to evaluate some design variations. The conclusion is that despite higher loss ratios, faster charging rates help the efficiency. The compensating winding helps reduce losses on the field coil and circumferentially narrow conductors are better than wider ones.

\section{FURTHER WORK}

The next level to which this model can be taken is to combine the finite element model with the entire external circuit including the converters and the launcher model. This will give an even more accurate representation of the internal dynamics of the compulsator. There will then be no need to have the data of a circuit simulation fed into the finite element analysis model.

\section{REFERENCES}

[1] I.A. Tsukerman, et a.l, "A method for circuit connections in time dependent eddy current problems”. IEEE Trans. on Magnetics, Vol. 28, No. 2, Mar. 1992 pgs. 1299-1302.

[2] P.M. Gresho and R.L. Lee, "Don't suppress the Wiggles - They're telling you something!” Lawrence Livermore Laboratory, University of California Livermore California, Report to the Department of Energy under contract no. W-7405-Eng-48.

[3] Bernard Adkins, The General Theory of Electrical Machines, John Wiley and Sons, 1957 pgs. 86-100.

[4] A.E. Fitzgerald and C. Kingsley, Electric Machinery, Second ed., McGraw Hill 1961, pgs 229-235.

TABLE 1

SUMMARY OF LOSS RATIOS FOR THE CONDITIONS INVESTIGATED (LOSS RATIO $=$ TRANSIENT LOSSES $/$ DC LOSSES $)$

\begin{tabular}{lccc}
\hline \hline \multicolumn{1}{c}{ Type of Design } & Charging & $\begin{array}{c}\text { Gun } \\
\text { Discharge }\end{array}$ & $\begin{array}{c}\text { Field } \\
\text { Reclaim }\end{array}$ \\
\hline Design with compensating winding & 1.39 & 1.085 & 1.332 \\
$\begin{array}{c}\text { As above but disabled } \\
\text { compensating winding }\end{array}$ & 1.4 & 1.2 & 1.35 \\
$\begin{array}{c}\text { Two layer - wider field } \\
\text { winding - no compensation winding }\end{array}$ & 2.04 & 1.4 & 1.75 \\
\hline \hline
\end{tabular}

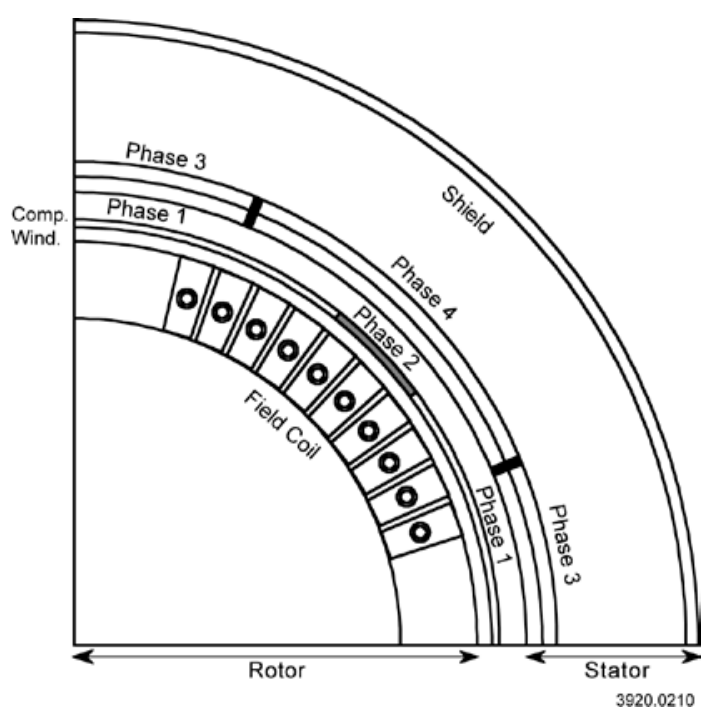

Fig. 1. Cross-section of the compulsator showing the various windings and the shield 

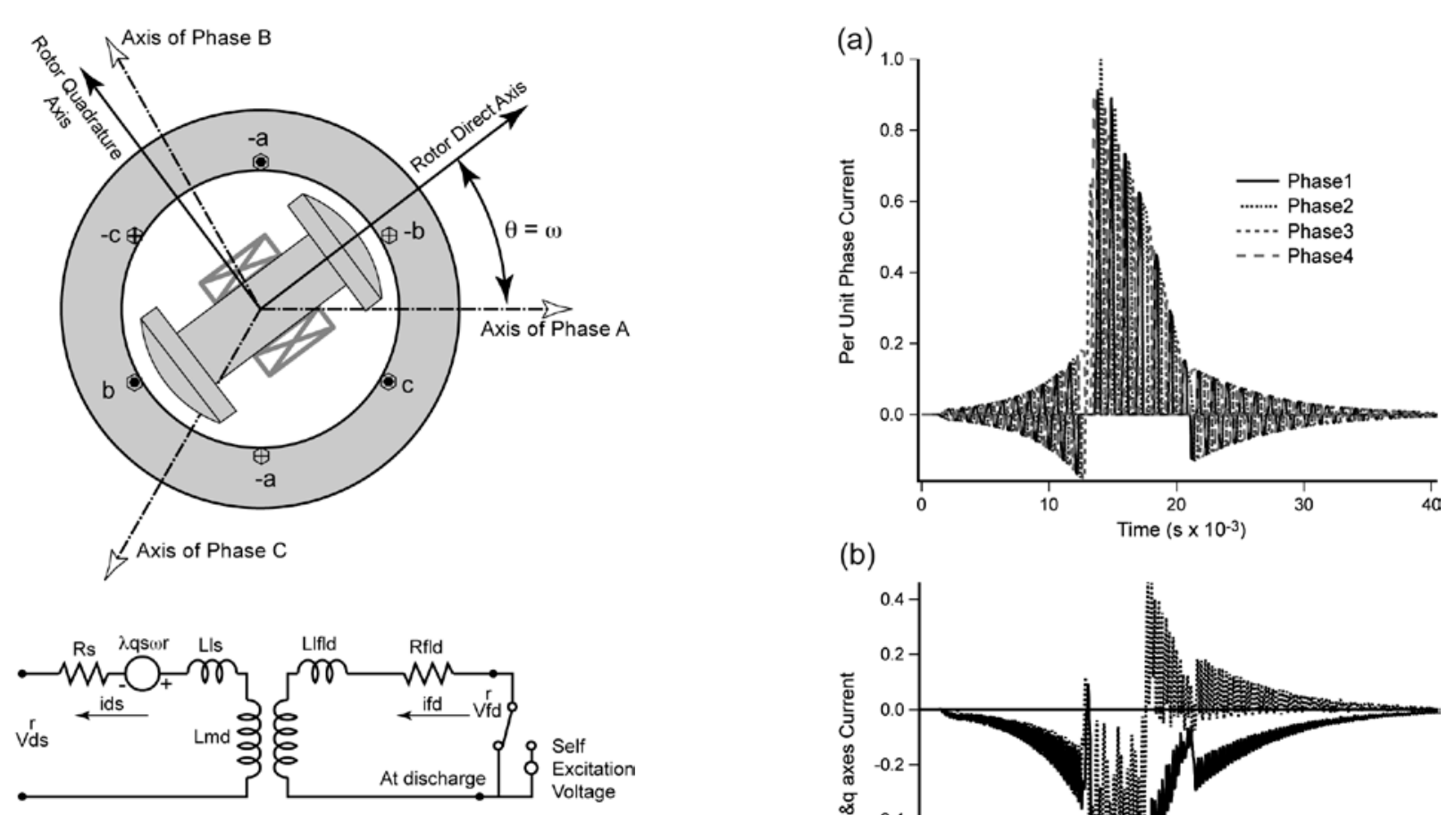

Equivalent Circuit with reference Axis on Rotor
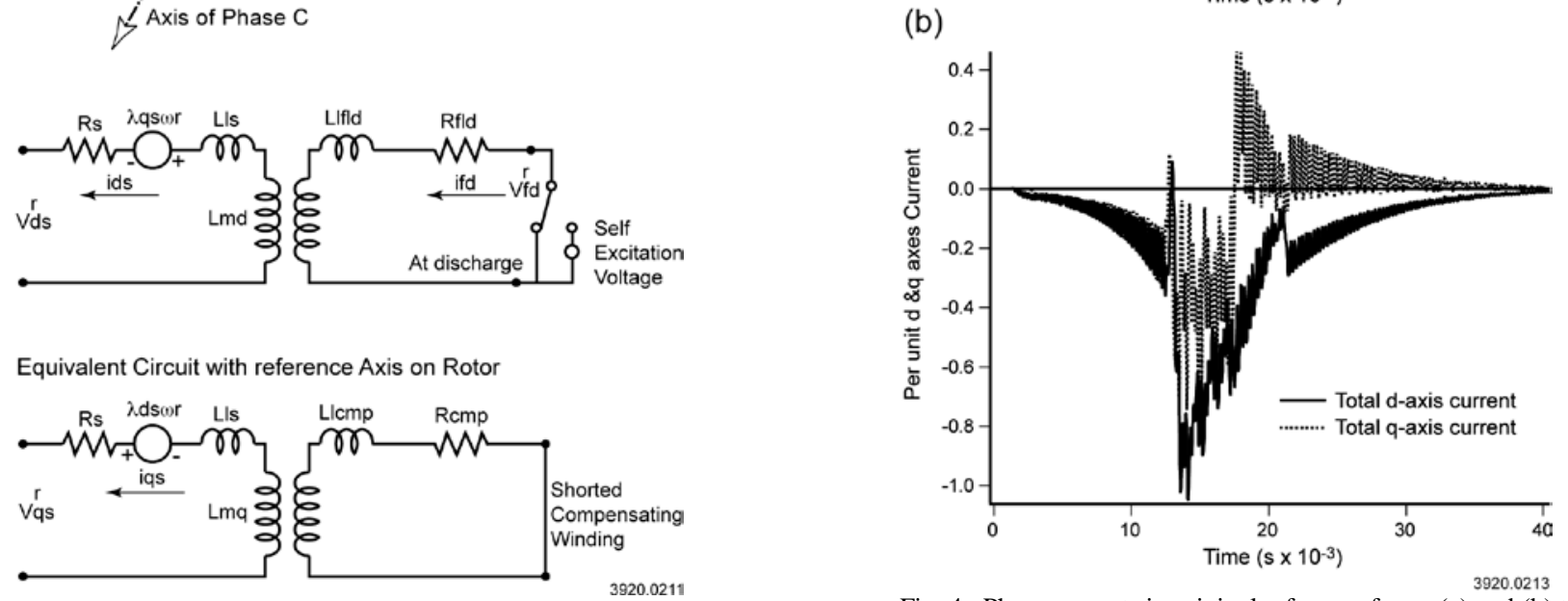

Fig. 2. Conventional machine showing the reference axes on the rotor and corresponding equivalent circuit

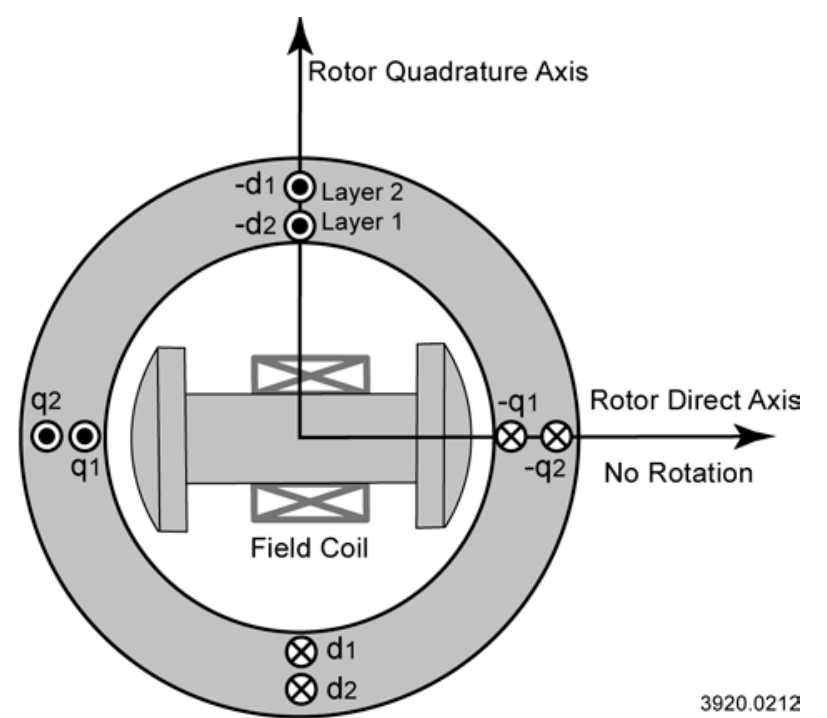

Fig. 4. Phase currents in original reference frame (a) and (b) total d and q axis currents in the transformed frame

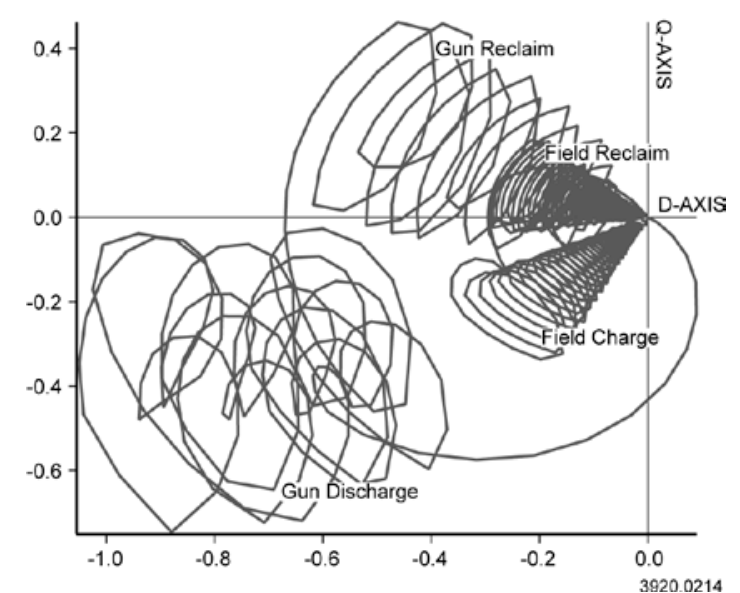

Fig. 5. Locus of points traversed by the stator magnetic axis during a typical discharge

Fig. 3. Stator windings referred to the rotor frame of reference (eliminating motional term) 


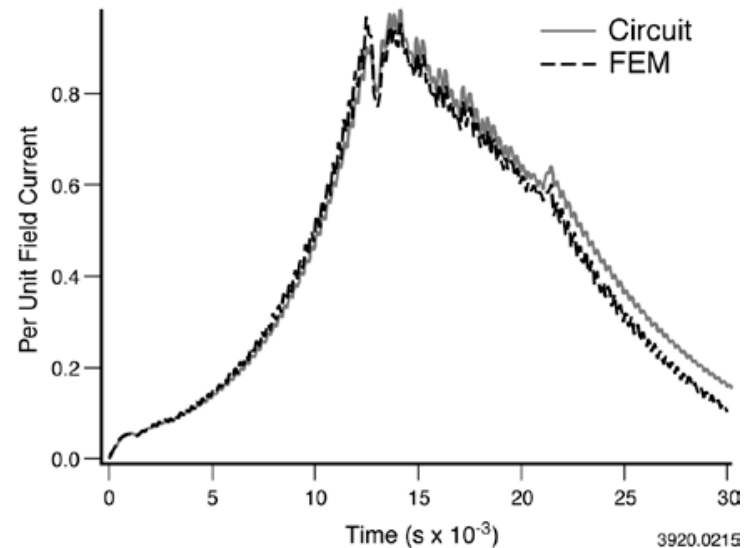

Fig. 6. Comparison of field current from circuit simulation and FEM model

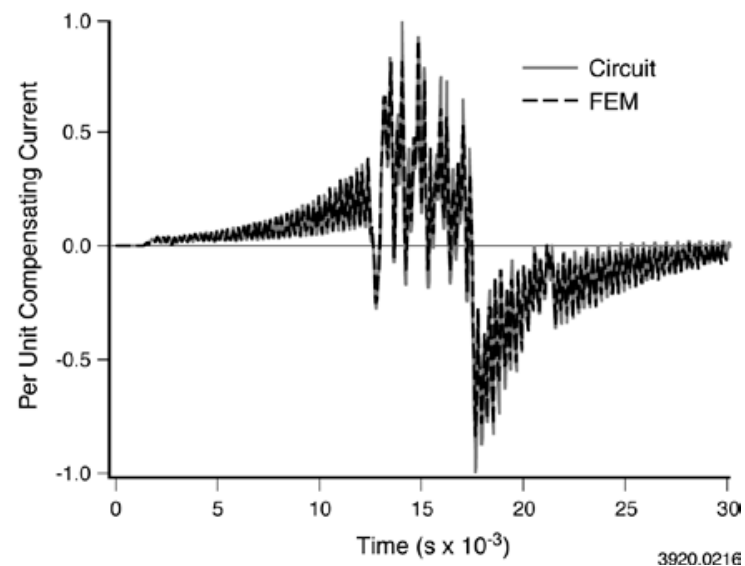

Fig. 7. Comparison of compensating current from circuit simulation and FEM model

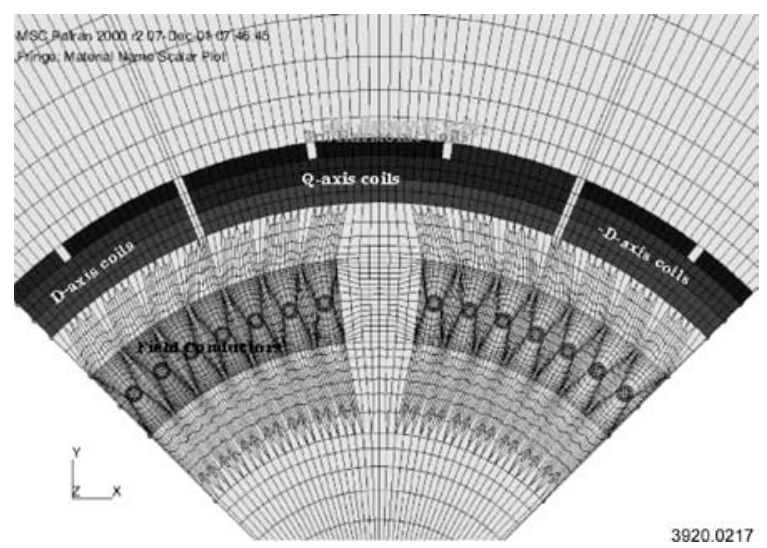

Fig. 8. The finite element model showing the different coils

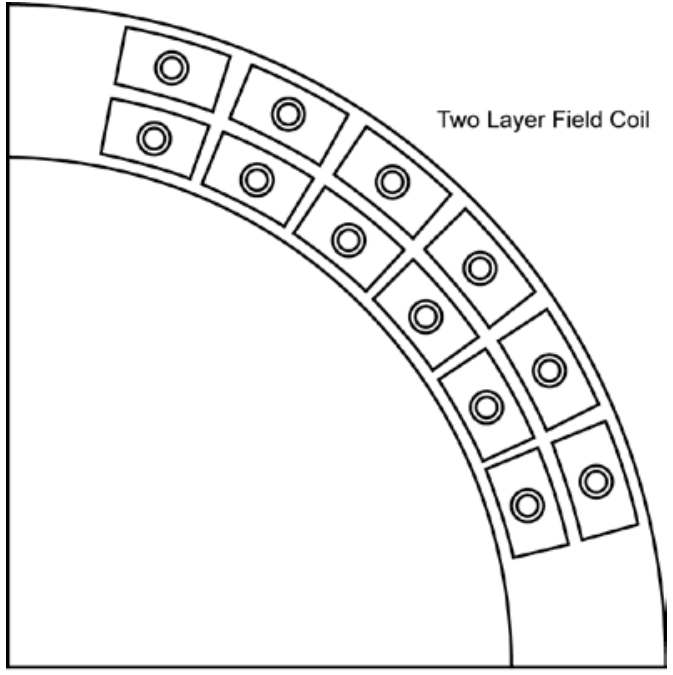

Fig. 9. Alternate \#2 layer field coil geometry

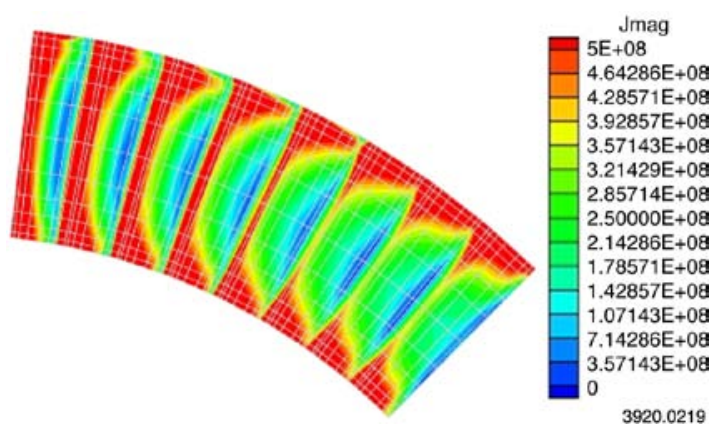

Fig. 10. Current distribution in the conductor at the end of charging with geometry (as in Fig. 1)

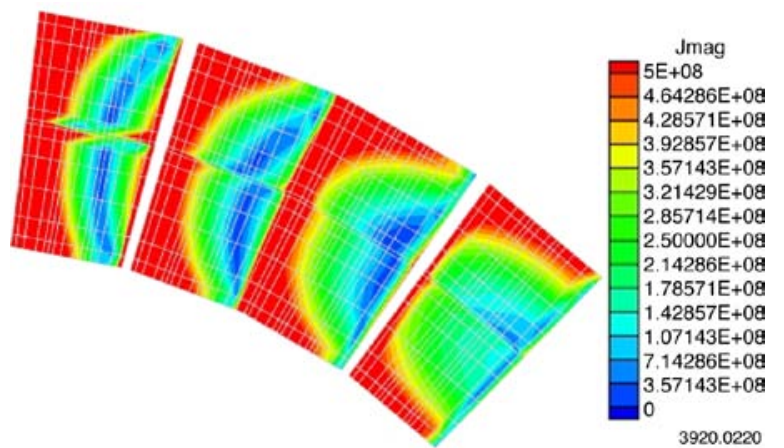

Fig. 11. Current distribution in the conductor at the end of charging with geometry (as in Fig. 8) 